\title{
Periumbilical Dermal Graft as a Good Option for Volume Enhancement in Secondary Cleft Lip Deformity: A Case Report
}

Seung Bum Pyo, Hong Sil Ju, Soo A Lim, Jin Kyung Song,

\section{Seong Yoon Lim}

Department of Plastic and Reconstructive Surgery, Hanil General Hospital, Seoul, Korea
Volume deficiency and poorly defined cupid's bow of the upper lip are frequently encountered problems in secondary cleft lip deformities. In this report, we present the method of a periumbilical dermal graft for correcting secondary cleft lip deformity. A 17-year-old male patient presented with a poorly defined notching cupid's bow and volume deficiency of the upper lip. He had undergone cleft lip surgery when he was 2 years old. We planned to perform a periumbilical dermal graft. There were sufficient amount of periumbilical subdermis and fat tissue thickness to obtain abundant volume. The periumbilical contour was similar to the natural contour of the lip. Less scarring was expected. There was no need to change the posture during operation, making it easy to harvest. Postoperative evaluations showed vermilion symmetry. Ideal cupid's bow shape and position were achieved without color mismatch. As a result, we obtained an ideal volume of the upper lip. Therefore a periumbilical dermal graft is a good option for correcting volume deficiency of the lip with good aesthetic outcomes.

Keywords Cleft lip, Dermis, Umbilicus
No potential conflict of interest relevant to this article was reported. deficiencies, and multiple treatments have been reported. One option is to supplement other tissues or use alloplastic material, to achieve a satisfactory operation result [2]. From the perspective of supplementation, various donors could be utilized. In this study, we would like to introduce an autologous periumbilical dermal graft as a good option for treating cleft lip deformity.

\section{CASE REPORT}

A 17-year-old Asian male patient presented with a poorly defined notching cupid's bow and volume deficiency of the upper lip (Fig. 1). He had undergone cleft lip surgery when he was 2 years old.

To correct the deformity, we evaluated and marked the amount of deficiency. The mid-line of face was located (Fig. 2). After designing, we performed excision of the previous scar following the minimally realigned excision line. We also excised the outer mucosa of the upper lip, and we performed mucosal dissection.

To have sufficient volume, we decided to harvest full layer of dermis and some dermal fat tissue in the periumbilical area (Fig. 3).

We harvested dermal graft with consideration of the recipient shape, tunneled between the muscle and mucosa of the upper lip 


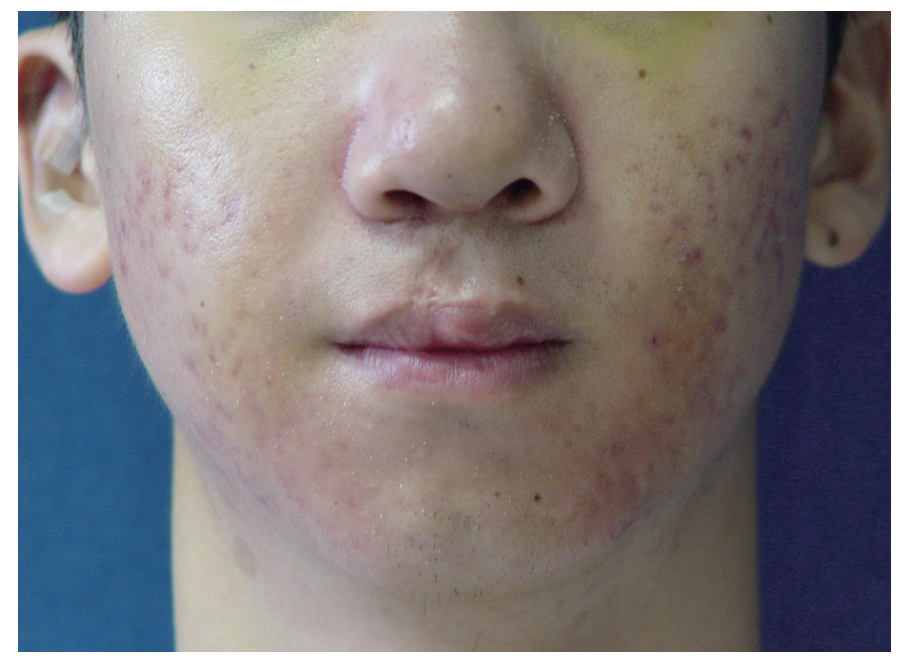

Fig. 1. Preoperative photo of patient showing deficiency of the cupid's bow and volume of the upper lip.

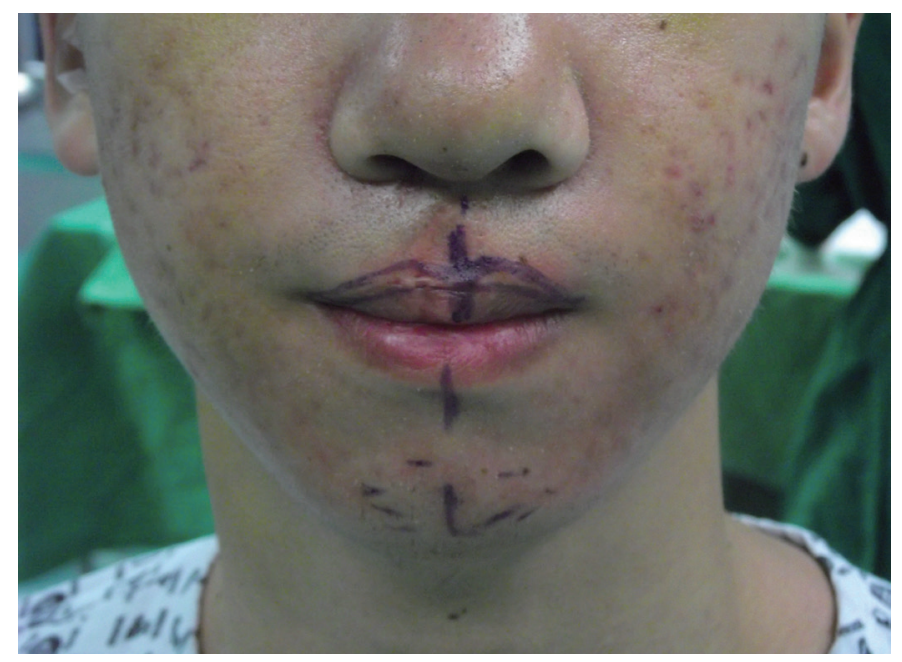

Fig. 2. Intraoperative photo of design.

to spare sufficient space (Fig. 4 and 5).

The graft was inserted into the submucosal pocket and fixed through pull-out suture on both lateral end (Fig. 6).

The operation result was evaluated by the surgeon based on cosmetic appearance, consistency of the upper lip vermilion and cupid's bow. The occurrence of complications, including infection and graft loss, was assessed during outpatient visit. Three months after the surgery, we observed a symmetric vermilions and ideal position of the cupid's bow (Fig. 7). Color inconsistency was not observed. Besides, the degree of firmness and stiffness assessed with Vancouver Scar Scale by the surgeon was adequate enough. We finally obtained an ideal amount of upper lip volume without complications such as infection or transplant rejection. The patient was satisfied with the results.

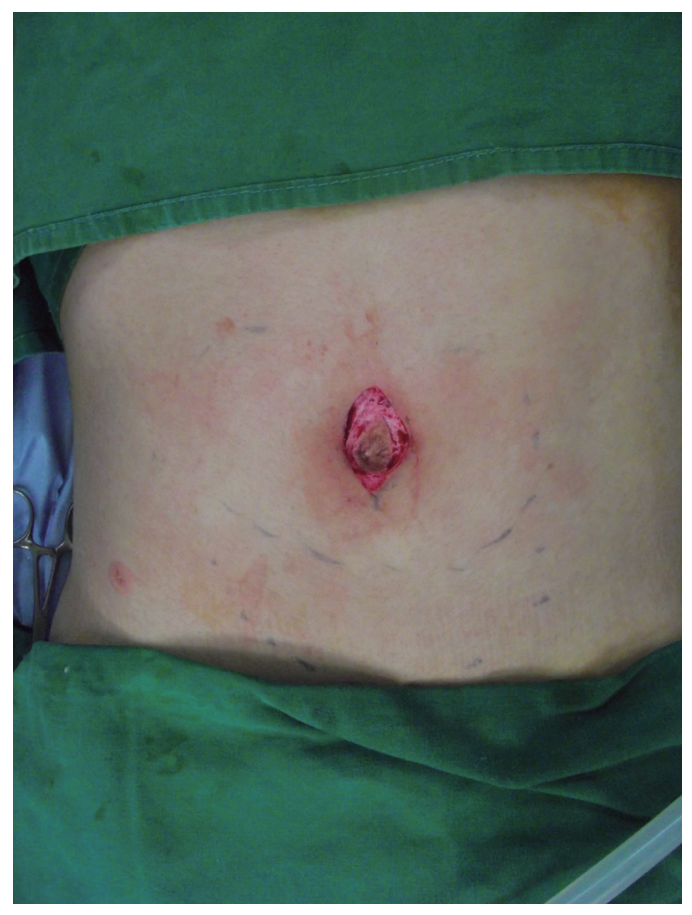

Fig. 3. Intraoperative photo of patient showing autologous croissant shape dermis graft harvested from the periumbilical area.

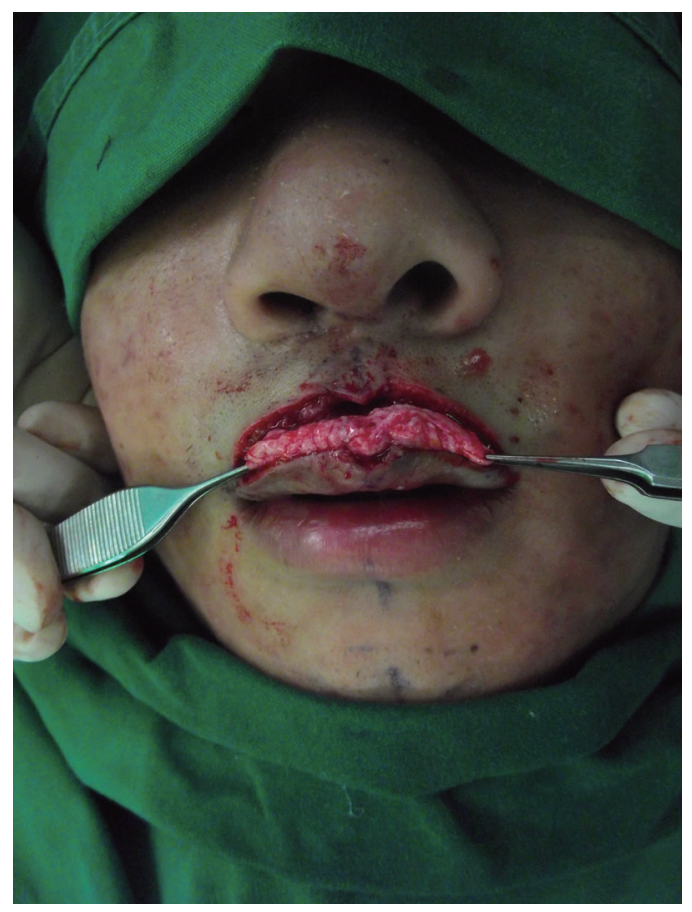

Fig. 4. Intraoperative photo of patient showing the dermis designed to fit the shape of the cupid's bow. It was inserted before mucosal dissection. 


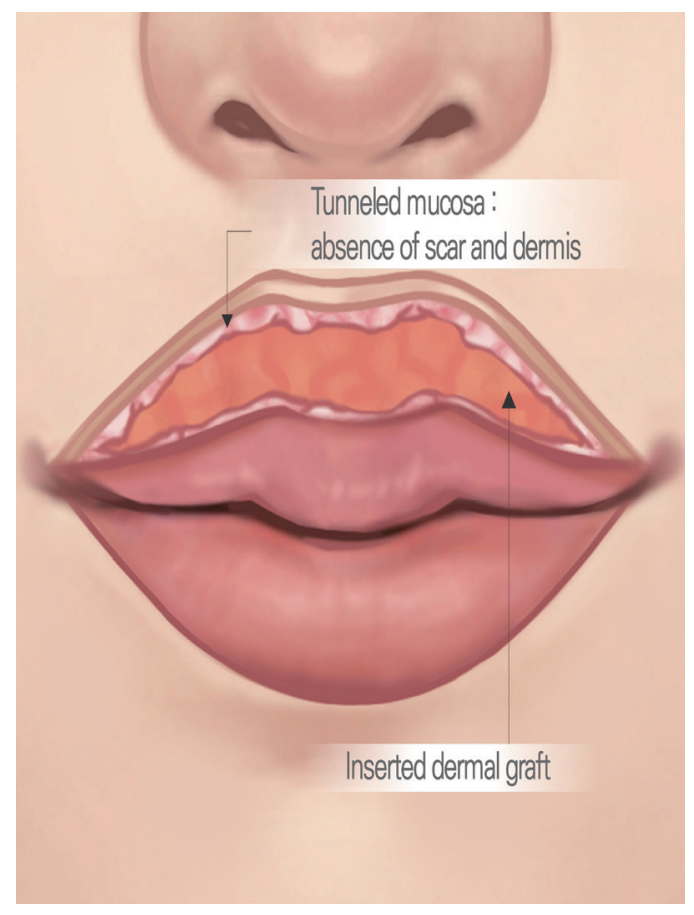

Fig. 5. The schematic image of the upper lip.

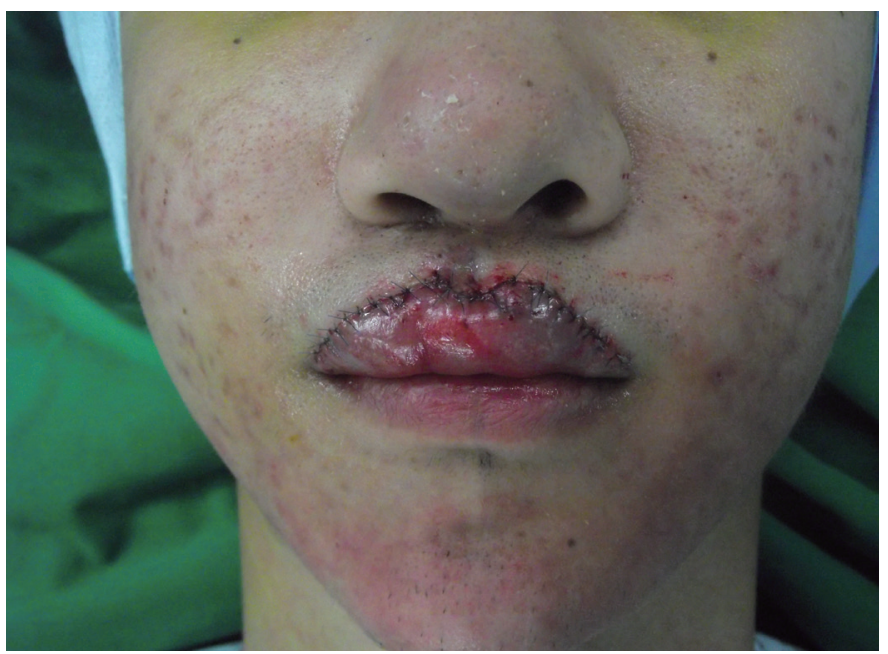

Fig. 6. Postoperative photo of patient showing the corrected upper lip after skin and the mucosal suture.

\section{DISCUSSION}

Secondary cleft lip deformities are usually accompanied by decreased lip volume and vermilion mismatch. Our study focused on the correction of secondary cleft lip deformity, where the periumbilical dermal graft has been utilized in various ways. Using this method, we were able to obtain a consistently sufficient volume of the upper lip. This technique can effectively be used for correction, as the end result looked similar to the natural lip.

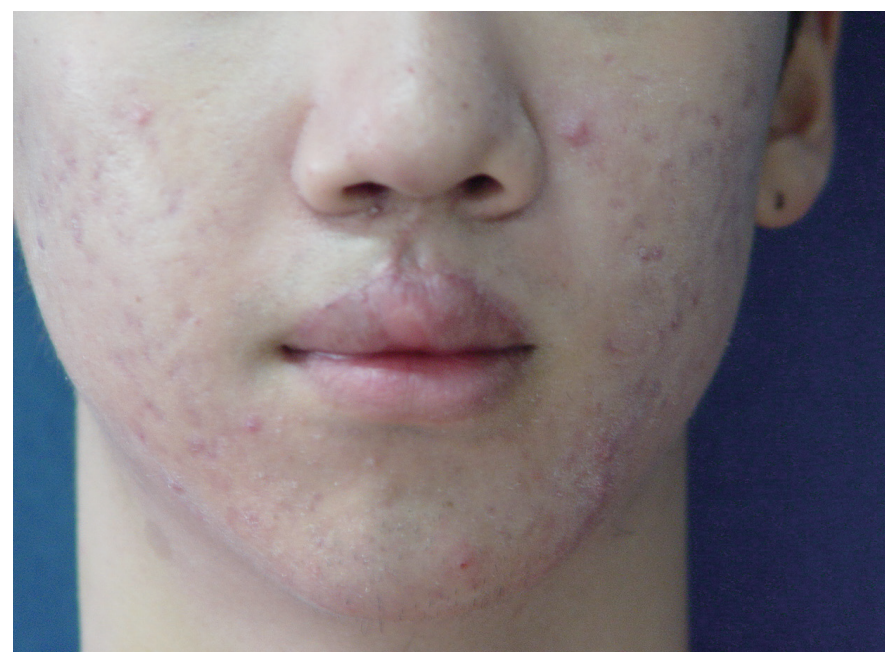

Fig. 7. Postoperative 3 months photo of patient showing deficiency of the cupid's bow. The volume of the upper lip was completely corrected.

As seen from our study, the periumbilical dermal graft has sufficient amount of volume, making it suitable for use in lip augmentation, and it can also be shaped in an appropriate manner as desired by the surgeon, making it a convenient operation. In addition, the periumbilical area is an extensively vascularized, relatively hairfree region, having a sufficiently thick dermis [3]. Being a non-exposed area, it leaves no noticeable skin scars, thus being cosmetically acceptable. The dermis of the lower abdomen is soft and elastic, and is almost impalpable after transplantation. If there is a requirement for a larger volume, the transplant site can be folded twice, and can be fixed with sophisticated catgut sutures or fibrin glue closure [4].

To date, many techniques have been described to correct the volume discrepancy of the upper lip in secondary correction of cleft lip deformity. The temporoparietal fascia has benefits not only in relevance to the cosmetic aspect, but also in functional aspect for lip augmentation, making it the most used technique. In addition, it exhibits a minimum graft resorption and shows a good prognosis in the long-term [1].

However, this technique has several disadvantages. The temporoparietal fascia needs to be incised at the subfollicular plane, for which the surgeon requires a certain extent of expertise, and the surgical procedure itself may be difficult. Also, since the temporoparietal fascia is thin and slippery, it can be difficult to obtain a sufficient amount [5]. Morbidity due to scarring alopecia formed on the scalp of the donor site has also been reported [2].

Alternately, the palmaris longus tendon has also been used for volume augmentation of the lip, and has shown improvements in terms of cosmetic and functional aspects. However, this method has the disadvantage wherein the scar remains, and there is a reduction of lip movements [6]. Thus, in surgeries of the secondary 
cleft lip deformity with a volume deficiency, there is a need for using other effective donor sites.

In this paper, we present a method to effectively manage cases of volume deficiency in secondary cleft lip deformity, through the periumbilical dermal graft. Here, we describe the effectiveness of the method, as it is possible to get enough volume needed for the transplant and a shape resembling the natural lip. In addition, similar to the temporoparietal fascia, it is not necessary to change positions during surgery, and is therefore more convenient than other donor sites. In future, the periumbilical dermal graft can be recommended as one of dermal graft sites to correct secondary cleft lip deformity due to lesser scaring on donor site, and good morbidity.

\section{PATIENT CONSENT}

Patients provided written consent for the use of their images.

\section{REFERENCES}

1. Chen PK, Noordhoff MS, Chen YR, et al. Augmentation of the free border of the lip in cleft lip patients using temporoparietal fascia. Plast Reconstr Surg 1995;95:781-8; discussion 9.

2. Lee KN, Koh KS. Acellular human dermis, a good option for correcting the free border deficiency in secondary cleft lip deformity. J Plast Reconstr Aesthet Surg 2012;65:356-61.

3. Bonavolontà G, Tranfa F, Salicone A, et al. Orbital dermis-fat graft using periumbilical tissue. Plast Reconstr Surg 2000;105:23-6.

4. Nicolle FV, Matti BA, Scamp T. Dermal and facial autografts in facial aesthetic surgery. Aesthetic Plast Surg 1992;16:219-25.

5. Thomas M, D'Silva J A, Chilgar RM. Volume enhancement of upper lip in secondary cleft deformity using composite mastoid tissue. Aesthetic Plast Surg 2012;36:414-20.

6. Trussler AP, Kawamoto HK, Wasson KL, et al. Upper lip augmentation: palmaris longus tendon as an autologous filler. Plast Reconstr Surg 2008;121:1024-32. 